\title{
Split common fixed point problem for Bregman demigener- alized mappings in Banach spaces with applications
}

\author{
Bashir Alia , G. C. Ugwunnadi ${ }^{b, *}$, M. S. Lawan ${ }^{c}$ \\ ${ }^{a}$ Department of Mathematical Sciences, Bayero University, Kano, Nigeria. \\ ${ }^{b}$ Department of Mathematics, University of Eswatini, Private Bag 4, Kwaluseni, Eswatini. \\ ${ }^{c}$ Department of Mathematics and Statistics, Kaduna Polytechnic, Kaduna, Nigeria.
}

\begin{abstract}
In this paper, we study the split common fixed point problem in reflexive Banach spaces, we obtain a strong convergence theorem for approximating a solution of the split common fixed point problem for Bregman demigeneralized mapping. Our result extend and improve important recent results announced by many authors.
\end{abstract}

Keywords: Bregman distance, Bregman demigeneralized mappings, split common fixed point problem, fixed point, Banach spaces.

2010 MSC: 47H09, 47J25.

(C)2020 All rights reserved.

\section{Introduction}

Let $E$ be a real reflexive Banach space with norm $\|\cdot\|$ and $E^{*}$ the dual space of $E$. Throughout this paper, we shall assume $f: E \rightarrow(-\infty,+\infty]$ is a proper, lower semi-continuous and convex function. We denote by $\operatorname{dom}(f):=\{x \in E: f(x)<+\infty\}$ the domain of $f$. Let $x \in \operatorname{int}(\operatorname{dom}(f))$; the subdifferential of $f$ at $x$ is the convex set defined by

$$
\partial f(x)=\left\{x^{*} \in E^{*}: f(x)+\left\langle x^{*}, y-x\right\rangle \leqslant f(y), \forall y \in E\right\},
$$

where the fenchel conjugate of $f$ is the function $f^{*}: E^{*} \rightarrow(-\infty,+\infty]$ defined by

$$
f^{*}\left(x^{*}\right)=\sup \left\{\left\langle x^{*}, x\right\rangle-f(x): x \in E\right\} .
$$

A function $f$ on $E$ is coercive [12] if the sublevel set of $f$ is bounded, equivalently,

$$
\lim _{\|x\| \rightarrow+\infty} f(x)=+\infty
$$

\footnotetext{
*Corresponding author

Email addresses: bashiralik@yahoo.com (Bashir Ali), ugwunnadi4u@yahoo.com (G. C. Ugwunnadi), mslawankh@yahoo.com (M.

S. Lawan)

doi: $10.22436 /$ jnsa.013.05.04
}

Received: 2019-10-31 Revised: 2020-01-23 Accepted: 2020-01-28 
A function $f$ on $E$ is said to be strongly coercive [27] if

$$
\lim _{\|x\| \rightarrow+\infty} \frac{f(x)}{\|x\|}=+\infty
$$

For any $x \in \operatorname{int}(\operatorname{dom}(f))$ and $y \in E$, the right-hand derivative of $f$ at $x$ in the direction of $y$ is defined by

$$
f^{\circ}(x, y):=\lim _{t \rightarrow 0^{+}} \frac{f(x+t y)-f(x)}{t} .
$$

The function $f$ is said to be Gâteaux differentiable at $x$ if $\lim _{t \rightarrow 0} \frac{f(x+t y)-f(x)}{t}$ exists for any $y$. In this case, the gradient of $f$ at $x$ is the function $\nabla f(x): E \rightarrow(-\infty,+\infty]$ defined by $\langle\nabla f(x), y\rangle=f^{\circ}(x, y)$ for any $y \in E$. The function $f$ is said to be Gâteaux differentiable if it is Gâteaux differentiable for any $x \in \operatorname{int}(\operatorname{dom}(f))$. The function $f$ is said to be Fréchet differentiable at $x$ if this limit is attained uniformly in $y,\|y\|=1$. The function $f$ is said to be uniformly Fréchet differentiable on a subset $C$ of $E$ if the limit is attained uniformly for $x \in C$ and $\|y\|=1$. It is well known that if $f$ is Gâteaux differentiable (resp. Fréchet differentiable) on $\operatorname{int}(\operatorname{dom}(f))$, then $f$ is continuous and its Gâteaux derivative $\nabla f$ is normto-weak* continuous (resp. norm-to-norm continuous) on $\operatorname{int}(\operatorname{dom}(f))($ see $[2,5])$. We will need the following results.

Lemma 1.1 ([18]). If $\mathrm{f}: \mathrm{E} \rightarrow \mathbb{R}$ is uniformly Fréchet differentiable and bounded on bounded subsets of $E$, then $\nabla \mathrm{f}$ is uniformly continuous on bounded subsets of $\mathrm{E}$ from the strong topology of $\mathrm{E}$ to the strong topology of $\mathrm{E}^{*}$.

Definition 1.2 ([4]). The function $f$ is said to be:

(1) essentially smooth, if $\partial f$ is both locally bounded and single-valued on its domain;

(ii) essentially strictly convex, if $(\partial f)^{-1}$ is locally bounded on its domain and $f$ is strictly convex on every subset of domf;

(iii) Legendre, if it is both essentially smooth and essentially strictly convex.

Remark 1.3. If $E$ is reflexive Banach space. Then we have the following results:

(i) $f$ is essentially smooth if and only if $f^{*}$ is essentially strictly convex (see [4] Theorem 5.4);

(ii) $(\partial f)^{-1}=\partial f^{*}($ see [5]);

(iii) $f$ is Legendre if and only if $f^{*}$ is Legendre (see [4, Corrolary 5.5]);

(iv) if $f$ is Legendre, then $\nabla f$ is a bijection satisfying $\nabla f=\left(\nabla f^{*}\right)^{-1}, \operatorname{ran} \nabla f=\operatorname{dom} \nabla\left(f^{*}\right)=\operatorname{int}\left(\operatorname{dom}\left(f^{*}\right)\right)$ and $\operatorname{ran} \nabla f^{*}=\operatorname{dom}(f)=\operatorname{int}(\operatorname{dom}(f))$ (see [4, Theorem 5.10]).

Examples of Legendre functions were given in $[3,4]$. One important and interesting Legendre function is $\frac{1}{p}\|\cdot\|^{p}(1<p<\infty)$ when $E$ is a smooth and strictly convex Banach space. In this case the gradient $\nabla f$ of $f$ is coincident with the generalized duality mapping of $E$, i.e, $\nabla f=J_{p}(1<p<\infty)$. In particular, $\nabla f=I$ the identity mapping in Hilbert spaces.

In the rest of this paper, we always assume that $f: E \rightarrow(-\infty,+\infty]$ is Legendre. Let $f: E \rightarrow(-\infty,+\infty]$ be a convex and Gateaux differentiable function. The function $D_{f}: \operatorname{dom} f \times \operatorname{intdomf} \rightarrow(-\infty,+\infty]$, defined as follows:

$$
D_{f}(x, y):=f(x)-f(y)-\langle\nabla f(y), x-y\rangle,
$$

is called the Bregman distance with respect to $f\left(\right.$ see [10] ). It is obvious from the definition of $D_{f}$ that

$$
D_{f}(z, x):=D_{f}(z, y)+D_{f}(y, x)+\langle\nabla f(y)-\nabla f(x), z-y\rangle .
$$

A point $p \in C$ is said to be asymptotic fixed point of a map $T$, if there exist a sequence $\left\{x_{n}\right\}$ in $C$ which converges weakly to $p$ such that $\lim _{n \rightarrow+\infty}\left\|x_{n}-T x_{n}\right\|=0$. We denote by $\hat{F}(T)$ the set of asymptotic 
fixed points of $T$. A point $p \in C$ is said to be strong asymptotic fixed point of a map $T$, if there exists a sequence $\left\{x_{n}\right\}$ in $C$ which converges strongly to $p$ such that $\lim _{n \rightarrow+\infty}\left\|x_{n}-T x_{\mathfrak{u}}\right\|=0$. We denote by $\tilde{F}(T)$ the set of strong asymptotic fixed points of $T$. A map $T: C \rightarrow C$ is called quasi-Bregman relatively nonexpansive [16] if $F(T) \neq \emptyset, \hat{F}(T)=F(T)$ and $D_{f}(T x, p) \leqslant D_{f}(x, p)$ for all $x \in C$ and $p \in F(T)$. T is said to be quasi-Bregman strictly pseudocontractive [26] if there exists a constant $\lambda \in[0,1)$ and $F(T) \neq \emptyset$ such that $D_{f}(p, T x) \leqslant D_{f}(p, x)+\lambda D_{f}(x, T x)$ for all $x \in C$ and $p \in F(T)$.

Let $E$ be a reflexive Banach space and $A: E \rightarrow 2^{E^{*}}$ multi-valued map, $A$ is said to be monotone if $\langle x-y, u-v\rangle \geqslant 0$ for all $x, y \in \operatorname{Dom}(A), u \in A x$ and $v \in A y$. A monotone operator $A$ is said to be maximal if the graph of $A$ is not properly contain in the graph of any other monotone operator on $E$.

Let $A$ be a maximal monotone mapping with $A^{-1}(0) \neq \emptyset$ and $f: E \rightarrow(-\infty,+\infty)$ be a uniformly Fréchet differentiable and bounded on bounded subsets of $E$, then the resolvent with $A$ and any $\lambda>0$ is defined by

$$
\operatorname{Res}_{A}^{f}(x)=(\nabla f+A)^{-1} \circ \nabla f(x)
$$

is single valued Bregman quasi-nonexpansive mapping from $E$ onto $D(A)$ and $F\left(\operatorname{Res}_{A}^{f}\right)=A^{-1}(0)$ (for details see [20]).

In 1967, Bregman [6] introduced an effective technique using the Bregman distance function $\mathrm{D}_{f}$ for designing and analyzing feasibility and optimization algorithms. This opened a growing area of research in which Bregman's technique is applied in various ways in order to design and analyze iterative algorithm for solving not only feasibility and optimization problems, but also algorithms for solving fixed point problems for nonlinear mappings (see, e.g., $[1,8,15,20,21,26]$ and the references therein).

Let $\mathrm{H}$ be a real Hilbert space and $\mathrm{C}$ a nonempty closed and convex subset of $\mathrm{H}$. A map T:C $\rightarrow \mathrm{H}$ is said to be inverse strongly monotone if there exists $\lambda>0$ such that

$$
\langle x-y, T x-T y\rangle \geqslant \lambda\|T x-T y\|^{2}, \quad \forall x, y \in C .
$$

Let $\mathrm{H}_{1}$ and $\mathrm{H}_{2}$ be real Hilbert spaces. Let $\mathrm{C}$ and $\mathrm{Q}$ be nonempty, closed and convex subsets of $\mathrm{H}_{1}$ and $\mathrm{H}_{2}$, respectively. Let $\mathrm{U}: \mathrm{H}_{1} \rightarrow \mathrm{H}_{2}$ be a bounded linear operator. Then the split feasibility problem is to find $z \in \mathrm{H}_{1}$ such that $z \in \mathrm{C} \cap \mathrm{U}^{-1} \mathrm{Q}$. Also let $\mathrm{A}: \mathrm{H}_{1} \rightarrow 2^{\mathrm{H}_{1}}$ and $\mathrm{B}: \mathrm{H}_{2} \rightarrow 2^{\mathrm{H}_{2}}$ be maximal monotone mappings, respectively, let $\mathrm{U}: \mathrm{H}_{1} \rightarrow \mathrm{H}_{2}$ be a bounded linear operator. The split common null point problem is to find $z \in \mathrm{H}_{1}$ such that $z \in A^{-1}(0) \cap \mathrm{U}^{-1} \mathrm{~B}^{-1}(0)$, where $A^{-1}(0)$ and $\mathrm{B}^{-1}(0)$ are the null point sets of $A$ and $B$, respectively. Given the mappings $\mathrm{T}: \mathrm{H}_{1} \rightarrow \mathrm{H}_{1}$ and $\mathrm{G}: \mathrm{H}_{2} \rightarrow \mathrm{H}_{2}$, respectively and $\mathrm{U}: \mathrm{H}_{1} \rightarrow \mathrm{H}_{2}$ bounded linear operator, the split common fixed point problem is to find $z \in H_{1}$ such that $z \in F(T) \cap A^{-1} F(G)$, where $F(T)$ and $F(G)$ are fixed point sets of $T$ and $G$, respectively. Let $M=U^{*}\left(I-P_{Q}\right) B$ be the split feasibility problem we have that $M: \mathrm{H}_{1} \rightarrow \mathrm{H}_{1}$ is an inverse strongly monotone operator where $\mathrm{U}^{*}$ is an adjoint operator of $\mathrm{U}$ and $\mathrm{P}_{\mathrm{Q}}$ is a metric projection of $\mathrm{H}_{2}$ onto $\mathrm{Q}$. Again if $\mathrm{C} \cap \mathrm{U}^{-1} \mathrm{Q}$ is nonempty then for $\lambda>0, z \in C \cap \mathrm{U}^{-1} \mathrm{Q}$ is equivalent to

$$
z=\mathrm{P}_{\mathrm{C}}\left(\mathrm{I}-\lambda \mathrm{U}^{*}\left(\mathrm{I}-\mathrm{P}_{\mathrm{Q}}\right) \mathrm{A}\right) z,
$$

where $P_{C}$ is a metric projection of $\mathrm{H}_{1}$ onto $C$. Also if $A^{-1}(0) \cap U^{-1} B^{-1}(0)$ is nonempty, then $z \in A^{-1}(0) \cap$ $\mathrm{U}^{-1} \mathrm{~B}^{-1}(0)$ is equivalent to

$$
z=J_{\lambda}\left(\mathrm{I}-\gamma \mathrm{U}^{*}\left(\mathrm{I}-\mathrm{Q}_{\mu}\right) \mathrm{U}\right) z,
$$

where $\gamma>0$ and $J_{\lambda}$ and $Q_{\mu}$ are resolvents of $A$ for $\lambda>0$ and $B$ for $\gamma>0$, respectively. Several authors have studied split feasibility problem, split common null point problem and split common fixed point problem (see, e.g., $[9,11,24]$ and the references therein). Hojo and Takahashi [13] extended (1.2) and (1.3) in Hilbert space to Banach space using the following iterative algorithm

$$
\left\{\begin{array}{l}
z_{n}=J_{\lambda_{n}}\left(x_{n}-\lambda_{n} T^{*} J_{F}\left(T x_{n}-Q_{\mu_{n}} T x_{n}\right)\right), \\
y_{n}=\alpha_{n} x_{n}+\left(1-\alpha_{n}\right) z_{n}, \\
C_{n+1}=\left\{z \in H:\left\|y_{n}-z\right\| \leqslant\left\|x_{n}-z\right\|\right\} \cap C_{n}, \\
x_{n+1}=P_{C_{n+1}} u_{n+1}, \quad n \in \mathbb{N},
\end{array}\right.
$$


under some appropriate conditions they proved that sequence $\left\{x_{n}\right\}$ converges strongly to a point $z \in$ $\mathrm{A}^{-1}(0) \cap \mathrm{T}^{-1} \mathrm{~B}^{-1}(0)$.

Let $E$ be a smooth Banach space and let $J$ be the duality mapping on $E$. The Lyapunov functional $\phi: E \times E \rightarrow \mathbb{R}$ is define by

$$
\phi(x, y)=\|x\|^{2}-2\langle x, J y\rangle+\|y\|^{2}, \quad \forall x, y \in E .
$$

Let $\eta$ and $s$ be real numbers with $\eta \in(-\infty, 1)$ and $s \in[0, \infty)$, respectively. Then the mapping $T: C \rightarrow E$ with $F(T) \neq \emptyset$ is called $(\eta, s)$-demigeneralized, if for any $x \in C$ and $q \in F(T)$,

$$
\langle x-\mathrm{q}, \mathrm{J} x-\mathrm{JT} x\rangle \geqslant(1-\eta) \phi(x, \mathrm{~T} x)+s \phi(T x, x),
$$

where $F(T)$ is the set of fixed points of $T$. In particular if $s=0$ in (1.4) then the mapping $T$ becomes

$$
\langle x-q, J x-J T x\rangle \geqslant(1-\eta) \phi(x, T x)
$$

for any $x \in C$ and $q \in F(T)$. $(\eta, 0)$-demigeneralized mappings are very important class of demigeneralized mappings.

Takahashi [23] studied the following iterative algorithm for solving split common null point problem and proved a strong convergence theorem for generalized resolvents in Banach spaces.

$$
\left\{\begin{array}{l}
z_{n}=J_{E}^{-1}\left(J_{E} x_{n}-r_{n} A^{*}\left(J_{F} T x_{n}-J_{F} Q_{\mu_{n}} A x_{n}\right)\right) \\
y_{n}=J_{\lambda_{n}} z_{n} \\
C_{n}=\left\{y \in E: 2\left\langle x_{n}-z, J_{E} x_{n}-J_{E} z_{n}\right\rangle \geqslant r_{n} \phi_{X}\left(A x_{n}, Q_{\mu_{n}} A x_{n}\right)\right\} \\
D_{n}=\left\{y \in E:\left\langle y_{n}-z, J_{E} z_{n}-J_{E} y_{n}\right\rangle \geqslant 0\right\} \\
Q_{n}=\left\{y \in E:\left\langle x_{n}-z, J_{E} x_{1}-J_{E} x_{n}\right\rangle \geqslant 0\right\} \\
x_{n+1}=\Pi_{C_{n} \cap D_{n} \cap Q_{n}} x_{1}, \quad n \geqslant 1,
\end{array}\right.
$$

under some mild conditions the sequence $\left\{x_{n}\right\}$ converges strongly to a point $z \in G^{-1}(0) \cap A^{-1} B^{-1}(0)$.

The following shrinking projection method was studied by Takahashi [22] for solving split common fixed point problem using demigeneralized mapping in Banach spaces

$$
\left\{\begin{array}{l}
z_{n}=J_{E}^{-1}\left(J_{E} x_{n}-r_{n} A^{*}\left(J_{F} A x_{n}-J_{F} U A x_{n}\right)\right) \\
y_{n}=T z_{n} \\
C_{n}=\left\{y \in E: 2\left\langle x_{n}-z_{1} J_{E} x_{n}-J_{E} z_{n}\right\rangle \geqslant r_{n}(1-\eta) \phi_{F}\left(A x_{n}, U A x_{n}\right)\right\} \\
D_{n}=\left\{y \in E: 2\left\langle y_{n}-z, J_{E} z_{n}-J_{E} y_{n}\right\rangle \geqslant(1-\tau) \phi_{E}\left(z_{n}, y_{n}\right)\right\} \\
Q_{n}=\left\{y \in E:\left\langle x_{n}-z, J_{E} x_{1}-J_{E} x_{n}\right\rangle \geqslant 0\right\} \\
x_{n+1}=\Pi_{C_{n} \cap D_{n} \cap Q_{n}} x_{1}, \quad n \geqslant 1,
\end{array}\right.
$$

under some appropriate conditions the sequence $\left\{x_{n}\right\}$ converges strongly to a point $z \in F(T) \cap A^{-1} F(U)$.

Motivated and inspired by the above mentioned results, we studied the split common fixed point problem in reflexive Banach spaces, we obtained a strong convergence theorem for approximating a solution of the split common fixed point problem for $(\eta, 0)$-Bregman demigeneralized mapping. Our result extends and improves the result of Takahashi [22] and many recently announced results.

\section{Preliminaries}

Recall that the Bregman projection [6] of $x \in$ intdomf onto nonempty, closed and convex set $C \subset \operatorname{domf}$ is the unique vector $P_{C}(x) \in C$ satisfying

$$
D_{f}\left(P_{K}(x), x\right)=\inf \left\{D_{f}(y, x): y \in C\right\} .
$$

Concerning the Bregman projection, the following are well known. 
Lemma 2.1 ([8]). Let $C$ be a nonempty, closed and convex subset of a reflexive Banach space $E$. Let $\mathrm{f}: \mathrm{E} \rightarrow \mathbb{R}$ be a Gâteaux differentiable and totally convex function and let $x \in \mathrm{E}$. Then

(b) $z=P_{K}(x)$ if and only if $\langle\nabla f(x)-\nabla f(z), y-z\rangle \leqslant 0, \quad \forall y \in C$;

(b) $D_{f}\left(y, P_{C}(x)\right)+D_{f}\left(P_{C}(x), x\right) \leqslant D_{f}(y, x), \quad \forall x \in E, y \in C$.

Let $f: E \rightarrow(-\infty,+\infty]$ be a Gâteaux differentiable function. The modulus of total convexity of $f$ at $x \in$ intdomf is the function $v_{f}(x, \cdot):[0,+\infty]$ defined by

$$
v_{f}(x, t):=\inf \left\{D_{f}(x, y): y \in \operatorname{domf},\|y-x\|=t\right\} .
$$

The function $f$ is called totally convex at $x$ if $v_{f}(x, t)>0$ whenever $t>0$. The function $f$ is called convex if it is totally convex at any point $x \in$ intdomf and is said to be totally convex on bounded set if $v_{f}(B, t)>0$ for any nonempty bounded subset $B$ of $E$ and $t>0$, where the modulus of total convexity of the function $f$ on the set $B$ is the function $v_{f}$ : intdomf $\times[0,+\infty) \rightarrow[0,+\infty)$ defined by

$$
v_{f}(B, t):=\inf \left\{v_{f}(x, t): x \in B \cap \operatorname{domf}\right\} .
$$

Lemma 2.2 ([17]). Let $\mathrm{E}$ be a Banach space and $\mathrm{f}: \mathrm{E} \rightarrow \mathbb{R}$ be a Gâteaux differentiable function which is uniformly convex on bounded subsets of $E$. Let $\left\{x_{n}\right\}_{n} \in \mathbb{N}$ and $\left\{y_{n}\right\}_{n \in \mathbb{N}}$ be bounded sequences in $E$. Then

$$
\lim _{n \rightarrow \infty} D_{f}\left(x_{n}, y_{n}\right)=0 \text { if and only if } \lim _{n \rightarrow \infty}\left\|x_{n}-y_{n}\right\|=0 \text {. }
$$

Lemma 2.3 ([19]). Let $\mathrm{f}: \mathrm{E} \rightarrow \mathbb{R}$ be Gâteaux differentiable and totally convex function. If $\mathrm{x}_{0} \in \mathrm{E}$ and the sequence $\left\{\mathrm{D}_{\mathrm{f}}\left(\mathrm{x}_{\mathrm{n}}, \mathrm{x}_{0}\right)\right\}$ is bounded the sequence $\left\{\mathrm{x}_{\mathrm{n}}\right\}$ is bounded too.

Recall that the function $f$ is called sequentially consistent if for any two sequences $\left\{x_{n}\right\}$ and $\left\{y_{n}\right\}$ in $E$ such that the first one is bounded

$$
\lim _{n \rightarrow+\infty} D_{f}\left(y_{n}, u_{n}\right)=0 \quad \text { implies } \quad \lim _{n \rightarrow+\infty}\left\|y_{n}-u_{n}\right\|=0 .
$$

Lemma 2.4 ([7]). The function $f$ is totally convex on bounded sets if and only if the function $f$ is sequentially consistent.

The following definition is slightly different from that in Butnariu and Iusem [7].

Definition 2.5 ([14]). Let $E$ be a Banach space. The function $f: E \rightarrow \mathbb{R}$ is said to be a Bregman function if the following conditions are satisfied:

(i) $f$ is continuous, strictly convex and Gâteaux differentiable;

(ii) the set $\left\{y \in E: D_{f}(x, y) \leqslant r\right\}$ is bounded for all $x \in E$ and $r>0$.

The following lemma follows from Butnariu and Iusem [7] and Zălinescu [27].

Lemma 2.6. Let $\mathrm{E}$ be a reflexive Banach space and $\mathrm{f}: \mathrm{E} \rightarrow \mathbb{R}$ be a strongly coercive Bregman function. Then

(i) $\nabla \mathrm{f}: \mathrm{E} \rightarrow \mathrm{E}^{*}$ is one-to-one, onto and norm-to-weak* continuous;

(ii) $\langle x-y, \nabla f(x)-\nabla f(y)\rangle=0$ if and only if $x=y$;

(iii) $\left\{x \in E: D_{f}(x, y) \leqslant r\right\}$ is bounded for all $y \in E$ and $r>0$;

(iv) dom $f^{*}=E^{*}, f^{*}$ is Gâteaux differentiable and $\nabla f^{*}=(\nabla f)^{-1}$.

The following two results are well known; see [27].

Theorem 2.7. Let $\mathrm{E}$ be a reflexive Banach space and let $\mathrm{f}: \mathrm{E} \rightarrow \mathbb{R}$ be a convex function which is bounded on bounded subsets of $\mathrm{E}$. Then the following assertions are equivalent:

(1) $f$ is strongly coercive and uniformly convex on bounded subsets of $E$; 
(2) domf* $=E^{*}, f^{*}$ is bounded on bounded subsets and uniformly smooth on bounded subsets of $E^{*}$;

(3) domf $f^{*}=E^{*}, f^{*}$ is Frechet differentiable and $\nabla f^{*}$ is norm-to-norm uniformly continuous on bounded subsets of $\mathrm{E}^{*}$.

Theorem 2.8. Let $\mathrm{E}$ be a reflexive Banach space and let $\mathrm{f}: \mathrm{E} \rightarrow \mathbb{R}$ be a continuous convex function which is strongly coercive. Then the following assertions are equivalent:

(1) $f$ is bounded on bounded subsets and uniformly smooth on bounded subsets of $E$;

(2) $f^{*}$ is Frechet differentiable and $f^{*}$ is uniformly norm-to-norm continuous on bounded subsets of $E^{*}$;

(3) domf* $=\mathrm{E}^{*}, \mathrm{f}^{*}$ is strongly coercive and uniformly convex on bounded subsets of $\mathrm{E}^{*}$.

Lemma 2.9 ([19]). Let $\mathrm{f}: \mathrm{E} \rightarrow \mathbb{R}$ be a Gâteaux differentiable and totally convex function, $\mathrm{x}_{0} \in \mathrm{E}$ and let $\mathrm{C}$ be a nonempty, closed and convex subset of $\mathrm{E}$. Suppose that the sequence $\left\{\mathrm{x}_{\mathrm{n}}\right\}$ is bounded and any weak subsequential limit of $\left\{x_{n}\right\}$ belongs to $C$. If $\mathrm{D}_{\mathrm{f}}\left(\mathrm{x}_{\mathrm{n}}, \mathrm{x}_{0}\right) \leqslant \mathrm{D}_{\mathrm{f}}\left(\mathrm{P}_{\mathrm{C}}\left(\mathrm{x}_{0}\right), \mathrm{x}_{0}\right)$ for any $\mathrm{n} \in \mathbb{R}$, then $\left\{\mathrm{x}_{\mathrm{n}}\right\}$ converges strongly to $\mathrm{P}_{\mathrm{C}}\left(\mathrm{x}_{0}\right)$.

Definition 2.10. Let $E$ be a reflexive Banach space, $C$ a nonempty closed and convex subset $E$, let $\eta$ be a real number with $\eta \in(-\infty, 1)$. Then the mapping $T: C \rightarrow E$ with $F(T) \neq \emptyset$ is called $(\eta, 0)$-Bregman demigeneralized mapping, if for any $x \in C$ and $q \in F(T)$,

$$
\langle x-q, \nabla f(x)-\nabla f(T x)\rangle \geqslant(1-\eta) D_{f}(x, T x),
$$

where $F(T)$ is the set of fixed points of $T$.

The following examples illustrate that the class of Bregman demigeneralized mappings are very important.

(i). [26] Let $C$ be a nonempty closed convex subset of reflexive Banach space $E$. Let $k$ be a real number in $(0,1)$, the map $T: C \rightarrow E$ is called quasi-Bregman strictly pseudocontractive mapping if $F(T) \neq \emptyset, \forall x \in C$ and $p \in F(T)$,

$$
D_{f}(p, T x) \leqslant D_{f}(p, x)+k D_{f}(x, T x),
$$

in fact $T$ is $(k, 0)$-Bregman demigeneralized mapping, from (1.1) and (2.1) we have,

$$
D_{f}(p, T x) \leqslant D_{f}(p, x)+D_{f}(x, T x)-D_{f}(x, T x)+k D_{f}(x, T x),
$$

which implies

$$
(1-k) D_{f}(x, T x) \leqslant D_{f}(p, x)+D_{f}(x, T x)-D_{f}(p, T x)=\langle x-p, \nabla f(x)-\nabla f(T x)\rangle .
$$

This shows that $\mathrm{T}$ is $(k, 0)$-Bregman demigeneralized mapping.

(ii).Let $E$ be a reflexive Banach space, let $f: E \rightarrow \mathbb{R}$ be strongly coercive function and let $A$ be a maximal monotone operator with $A^{-1} \neq \emptyset$. Let $\lambda>0 \operatorname{Res}_{\lambda A}^{f}$ is $(0,0)$-Bregman demigeneralized mapping, for any $x \in E$ and $z \in A^{-1}(0)$ we have

$$
\left\langle\operatorname{Res}_{\lambda A}^{f}(x)-z, \nabla f(x)-\nabla f\left(\operatorname{Res}_{\lambda A}^{f}(x)\right)\right\rangle \geqslant 0,
$$

which gives

$$
\left\langle\operatorname{Res}_{\lambda A}^{f}(x)-x+x-z, \nabla f(x)-\nabla f\left(\operatorname{Res}_{\lambda A}^{f}(x)\right)\right\rangle \geqslant 0,
$$

hence,

$$
\begin{aligned}
\left\langle x-z, \nabla f(x)-\nabla f\left(\operatorname{Res}_{\lambda A}^{f}(x)\right)\right\rangle & \geqslant\left\langle x-\operatorname{Res}_{\lambda A}^{f}(x), \nabla f(x)-\nabla f\left(\operatorname{Res}_{\lambda A}^{f}(x)\right)\right\rangle \\
& =D_{f}\left(x, \operatorname{Res}_{\lambda A}^{f}(x)\right)+D_{f}\left(\operatorname{Res}_{\lambda A}^{f}(x), x\right) \geqslant D_{f}\left(x, \operatorname{Res}_{\lambda A}^{f}(x)\right) .
\end{aligned}
$$

This shows that $\operatorname{Res}_{\lambda A}^{f}$ is $(0,0)$-Bregman demigeneralized mapping. 
(iii).Let $E$ be a reflexive Banach space and $C$ a nonempty closed convex subset of $E$ and let $f: E \rightarrow \mathbb{R}$ be a Fréchet differentiable convex function. A map $T: C \rightarrow E$ is called quasi-Bregman nonexpansive mapping if $F(T) \neq \emptyset$ and for all $x \in C, p \in F(T)$,

$$
D_{f}(p, T x) \leqslant D_{f}(p, x),
$$

then $T$ is $(0,0)$-Bregman demigeneralized mapping. For all $x \in C, p \in F(T)$ we have

$$
D_{f}(p, T x) \leqslant D_{f}(p, x),
$$

which by (1.1) we get

$$
D_{f}(p, x)+D_{f}(x, T x)+\langle p-x, \nabla f(x)-\nabla f(T x)\rangle \leqslant D_{f}(p, x)
$$

and hence

$$
D_{f}(x, T x) \leqslant\langle x-p, \nabla f(x)-\nabla f(T x)\rangle
$$

This implies that $\mathrm{T}$ is $(0,0)$-Bregman demigeneralized mapping.

Example 2.11. Let $E=\mathbb{R}, C=[-1,0]$ and define $T, f:[-1,0] \rightarrow \mathbb{R}$ by $f(x)=x^{3}$ and $T x=2 x$, for all $x \in[-1,0]$. Then $T$ is $(\eta, 0)$-Bregman demigeneralized mapping but not $(\eta, 0)$-demigeneralized mapping.

Proof. It is clear from the definition that $\mathrm{f}$ is proper, lower semi-continuous, and convex, also $\mathrm{F}(\mathrm{T})=\{0\}$. From the definition $(\eta, 0)$-Bregman demigeneralized mapping, we have for any $\eta \in(-\infty, 1)$ such that

$$
\langle x-q, \nabla f(x)-\nabla f(T x)\rangle \geqslant(1-\eta) D_{f}(x, T x) \text { for all } x \in C, q \in F(T) .
$$

But

$$
\begin{aligned}
\langle x-0, \nabla f(x)-\nabla f(T x)\rangle & =\langle x-0, \nabla f(x)-\nabla f(2 x)\rangle \\
& =\left\langle x, \nabla\left(x^{3}\right)-\nabla\left(8 x^{3}\right)\right\rangle \\
& =\left\langle x, 3 x^{2}-24 x^{2}\right\rangle \\
& =\left\langle x,-21 x^{2}\right\rangle=-21 x^{3}
\end{aligned}
$$

and

$$
\begin{aligned}
D_{f}(x, T x) & =f(x)-f(T x)-\langle\nabla f(T x), x-T x\rangle \\
& =x^{3}-f(2 x)-\left\langle 24 x^{2}, x-2 x\right\rangle \\
& =x^{3}-8 x^{3}-\left\langle 24 x^{2},-x\right\rangle \\
& =-7 x^{3}+24 x^{3}=17 x^{3} .
\end{aligned}
$$

Therefore from (2.2) and (2.3) we have

$$
\begin{aligned}
\langle x-0, \nabla f(x)-\nabla f(T x)\rangle & =-21 x^{3} \\
& \geqslant(1-\eta) 17 x^{3}, \quad \forall x \in[-1,0], \eta \in(-\infty, 1) \\
& \geqslant(1-\eta) D_{f}(x, T x), \quad \forall x \in[-1,0], \eta \in(-\infty, 1), \\
-21 x^{3} & \geqslant(1-\eta) 17 x^{3} \Longrightarrow \quad 21=(1-\eta) 17 \Longrightarrow(1-\eta)=\frac{21}{17} \Longrightarrow \quad \eta=\frac{4}{17}
\end{aligned}
$$

Therefore

$$
\langle x-0, \nabla f(x)-\nabla f(T x)\rangle \geqslant(1-\eta) D_{f}(x, T x), \quad \forall x \in[-1,0], \eta \in(-\infty, 1) .
$$


Hence $T$ is $(\eta, 0)$-Bregman demigeneralized mapping. Further

$$
\begin{aligned}
\langle x-0, \mathrm{Jx}-\mathrm{JT} x\rangle & =\langle x-0, \mathrm{~J}(\mathrm{x})-\mathrm{J}(2 x)\rangle \\
& =\langle x, \mathrm{~J}(x)\rangle-\langle x, \mathrm{~J}(2 x)\rangle \\
& =|x|^{2}-2|x|^{2}=-|x|^{2}
\end{aligned}
$$

and

$$
\begin{aligned}
\phi(x, T x) & =|x|^{2}-2\langle x, J(T x)\rangle+|T x|^{2} \\
& =|x|^{2}-2\langle x, J(2 x)\rangle+|2 x|^{2} \\
& =|x|^{2}-4\langle x, J(x)\rangle+4|x|^{2} \\
& =|x|^{2}-4|x|^{2}+4|x|^{2}=|x|^{2} .
\end{aligned}
$$

Since $-|x|^{2}<(1-\eta)|x|^{2}, \forall x \in[-1,0]$, and for all $\eta \in(-\infty, 1)$,

$$
\langle x-0, \mathrm{~J} x-\mathrm{JT} x\rangle \geqslant(1-\eta) \phi(x, T x), \quad \forall x \in[-1,0],
$$

can not hold for any $\eta \in(-\infty, 1)$. Hence $T$ is not a demigeneralized mapping.

Assumption 2.12. Let $\mathrm{E}$ be a reflexive Banach space and $\mathrm{C}$ a nonempty closed convex subset of $\mathrm{E}$. A mapping $\mathrm{T}: \mathrm{C} \rightarrow \mathrm{E}$ is called demiclosed if for any sequence $\left\{\mathrm{x}_{\mathrm{n}}\right\} \in \mathrm{C}$ such that $\mathrm{x}_{\mathrm{n}} \rightarrow \mathrm{p}$ and $\mathrm{x}_{\mathrm{n}}-\mathrm{T} \mathrm{x}_{\mathrm{n}} \rightarrow 0$, then $\mathrm{p}=\mathrm{Tp}$ holds.

\section{Main result}

Lemma 3.1. Let $\mathrm{E}$ be a reflexive Banach space and $\mathrm{C}$ a nonempty closed and convex subset of $\mathrm{E}$. Let $\mathrm{f}: \mathrm{E} \rightarrow \mathbb{R}$ be a strongly coercive, Legendre function, which is bounded, uniformly Fréchet differentiable and totally convex on bounded subset of $\mathrm{E}$. Let $\eta$ be a real number with $\eta \in(-\infty, 1)$ and $\mathrm{T}$ an $(\eta, 0)$-Bregman demigeneralized mapping of $\mathrm{C}$ onto $\mathrm{E}$. Then $\mathrm{F}(\mathrm{T})$ is closed and convex.

Proof. We first show that $F(T)$ is closed. For a sequence $\left\{q_{n}\right\}$ in $F(T)$ such that $q_{n} \rightarrow q$ and in $C$, we have from the definition of $T$ that

$$
\left\langle q-q_{n}, \nabla f(q)-\nabla f(T q)\right\rangle \geqslant(1-\eta) D_{f}(q, T q),
$$

from $q_{n} \rightarrow q$ we have $0 \geqslant(1-\eta) D_{f}(q, T q)$. From $1-\eta>0$, we get $0 \geqslant D_{f}(q, T q)$ and hence $0=D_{f}(q, T q)$. Since $E$ is reflexive, we have $q=T q$. This implies that $F(T)$ is closed. Secondly we show that $F(T)$ is convex. Let $p, q \in F(T)$ and set $x=\alpha p+(1-\alpha) q$, where $\alpha \in[0,1]$. From the definition of $T$ we have that for $x \in C$ and $q \in F(T)$,

$$
\langle x-q, \nabla f(x)-\nabla f(T x)\rangle \geqslant(1-\eta) D_{f}(x, T x) .
$$

This implies from (1.1) that

$$
D_{f}(q, x)+D_{f}(x, T x)-D_{f}(q, T x) \geqslant(1-\eta) D_{f}(x, T x),
$$

which implies

$$
D_{f}(q, x)+D_{f}(x, T x)-D_{f}(x, T x)+\eta D_{f}(x, T x) \geqslant D_{f}(q, T x)
$$

and hence

$$
D_{f}(q, x)+\eta D_{f}(x, T x) \geqslant D_{f}(q, T x) .
$$

Using this, we have for $x=\alpha p+(1-\alpha) q$, and $p, q \in F(T)$

$$
D_{f}(x, T x)=f(x)-f(T x)-\langle\nabla f(T x), x-T x\rangle
$$




$$
\begin{aligned}
= & f(x)-f(T x)-\langle\nabla f(T x),(\alpha p+(1-\alpha) q)-T x\rangle \\
= & f(x)-f(T x)-\alpha\langle\nabla f(T x), p-T x\rangle-(1-\alpha)\langle\nabla f(T x), q-T x\rangle \\
= & f(x)+\alpha f(p)-\alpha f(T x)-\alpha\langle\nabla f(T x), p-T x\rangle \\
& +(1-\alpha) f(q)-(1-\alpha) f(T x)-(1-\alpha)\langle\nabla f(T x), q-T x\rangle-\alpha f(p)-(1-\alpha) f(q) \\
= & f(x)+\alpha D_{f}(p, T x)+(1-\alpha) D_{f}(q, T x)-f(x) \\
\leqslant & \alpha\left(D_{f}(p, x)+\eta D_{f}(x, T x)\right)+(1-\alpha)\left(D_{f}(q, x)+\eta D_{f}(x, T x)\right) \\
= & \alpha f(p)-\alpha f(x)-\alpha\langle\nabla f(x), p-x\rangle+\alpha \eta D_{f}(x, T x) \\
& +(1-\alpha) f(q)-(1-\alpha) f(x)-(1-\alpha)\langle\nabla f(x), q-x\rangle+(1-\alpha) \eta D_{f}(x, T x) \\
= & \left.\alpha f(p)+(1-\alpha) f(q)-f(x)-\langle\nabla f(x),(\alpha p+(1-\alpha) q)-x\rangle+\eta D_{f}(x, T x)\right) \\
= & \eta D_{f}(x, T x),
\end{aligned}
$$

this implies $0 \leqslant(\eta-1) D_{f}(x, T x)$ we have from the fact $0>(\eta-1)$ that $D_{f}(x, T x)=0$ and from Lemma 7.3 in [4] it follows that $x=T x$. Therefore $F(T)$ is convex.

Theorem 3.2. Let $\mathrm{E}$ and $\mathrm{X}$ be reflexive Banach spaces, $\mathrm{f}: \mathrm{E} \rightarrow \mathbb{R}$ and $\mathrm{g}: \mathrm{X} \rightarrow \mathbb{R}$ be strongly coercive Legendre functions which are bounded uniformly Fréchet differentiable and totally convex on bounded subsets of $E$ and $X$, respectively. Let $\eta$ and $\tau$ be real numbers with $\eta, \tau \in(-\infty, 1)$. Let $G: E \rightarrow E$ be $(\eta, 0)$-Bregman demigeneralized mapping with $\mathrm{F}(\mathrm{G}) \neq \emptyset$ and let $\mathrm{T}: \mathrm{X} \rightarrow \mathrm{X}$ be $(\tau, 0)$-Bregman demigeneralized mapping with $\mathrm{F}(\mathrm{T}) \neq \emptyset$. Let $\mathrm{B}: \mathrm{E} \rightarrow \mathrm{X}$ be a bounded linear operator such that $\|\mathrm{B}\| \neq 0$ and let $\mathrm{B}^{*}$ be the adjoint operator of $\mathrm{B}$. Suppose $\Omega=\mathrm{F}(\mathrm{G}) \cap \mathrm{B}^{-1} \mathrm{~F}(\mathrm{~T}) \neq \emptyset$. Let $\left\{\mathrm{x}_{\mathrm{n}}\right\}$ be a sequence generated by the following algorithm and $\mathrm{x}_{1} \in \mathrm{E}$

$$
\left\{\begin{array}{l}
z_{\mathfrak{n}}=\nabla f^{*}\left(\nabla f\left(x_{n}\right)-r_{n} B^{*}\left(\nabla g\left(B x_{n}\right)-\nabla g\left(T B x_{n}\right)\right)\right) \\
y_{n}=G z_{n} \\
C_{n}=\left\{y \in E:\left\langle x_{n}-y, \nabla f\left(x_{n}\right)-\nabla f\left(z_{n}\right)\right\rangle \geqslant r_{n}(1-\eta) D_{g}\left(B x_{n}, T B x_{n}\right)\right\} \\
D_{n}=\left\{y \in E:\left\langle z_{n}-y, \nabla f\left(z_{n}\right)-\nabla f\left(y_{n}\right)\right\rangle \geqslant(1-\tau) D_{f}\left(z_{n}, y_{n}\right)\right\} \\
Q_{n}=\left\{y \in E:\left\langle x_{n}-y, \nabla f\left(x_{1}\right)-\nabla f\left(x_{n}\right)\right\rangle \geqslant 0\right\} \\
x_{n+1}=P_{C_{n} \cap D_{n} \cap Q_{n}}^{f} x_{1}, \quad n \geqslant 1,
\end{array}\right.
$$

where $r_{n} \subset(0, \infty)$ and $a \in(0, \infty)$ satisfying the following inequalities

$$
0<\mathrm{a} \leqslant \mathrm{r}_{\mathrm{n}} \leqslant \frac{1}{\|\mathrm{~B}\|^{2}}, \quad \forall \mathrm{n} \in \mathbb{N} .
$$

Then $\left\{x_{n}\right\}$ converges strongly to $p \in \Omega$ where $p=\mathrm{P}_{\Omega}^{\mathrm{f}} \mathrm{x}_{1}$.

Proof. It is easy to see that $C_{n} \cap D_{n} \cap Q_{n}$ is closed and convex for all $n \geqslant 1$. To show $\Omega \in C_{n}$ for all $n \geqslant 1$, we need to show that

$$
\left\langle x_{n}-p, \nabla f\left(x_{n}\right)-\nabla f\left(z_{n}\right)\right\rangle \geqslant r_{n}(1-\eta) D_{g}\left(B x_{n}, T B x_{n}\right)
$$

for all $p \in B^{-1} F(T)$ and $n \geqslant 1$. Thus we have

$$
\begin{aligned}
\left\langle x_{n}-p, \nabla f\left(x_{n}\right)-\nabla f\left(z_{n}\right)\right\rangle & =r_{n}\left\langle x_{n}-p, B^{*}\left(\nabla g\left(B x_{n}\right)-\nabla g\left(T B x_{n}\right)\right)\right\rangle \\
& =r_{n}\left\langle B x_{n}-B p, \nabla g\left(B x_{n}\right)-\nabla g\left(T B x_{n}\right)\right\rangle \\
& \geqslant r_{n}(1-\eta) D_{g}\left(B x_{n}, T B x_{n}\right) .
\end{aligned}
$$

This shows $\Omega \subset B^{-1} F(T) \subset C_{n}$ for all $n \geqslant 1$. We next show that $\Omega \subset D_{n}$ for all $n \geqslant 1$, we show that

$$
\left\langle z_{n}-p, \nabla f\left(z_{n}\right)-\nabla f\left(y_{n}\right)\right\rangle \geqslant(1-\tau) D_{f}\left(z_{n}, y_{n}\right)
$$


for all $p \in F(G)$ and $n \geqslant 1$. Thus we have

$$
\begin{aligned}
\left\langle z_{n}-p, \nabla f\left(z_{n}\right)-\nabla f\left(y_{n}\right)\right\rangle-(1-\tau) D_{f}\left(z_{n}, y_{n}\right) & =\left\langle z_{n}-p, \nabla f\left(z_{n}\right)-\nabla f\left(G z_{n}\right)\right\rangle-(1-\tau) D_{f}\left(z_{n}, G z_{n}\right) \\
& \geqslant(1-\tau)\left(D_{f}\left(z_{n}, G z_{n}\right)-D_{f}\left(z_{n}, G z_{n}\right)\right)=0 .
\end{aligned}
$$

This implies that $\Omega \subset F(G) \subset D_{n}$ for all $n \geqslant 1$. We now show that $\Omega \subset Q_{n}$ for all $n \geqslant 1$. From (3.1) we have

$$
\mathrm{Q}_{1}=\left\{\mathrm{p} \in \mathrm{E}:\left\langle\mathrm{x}_{1}-\mathrm{p}, \nabla \mathrm{f}\left(\mathrm{x}_{1}\right)-\nabla \mathrm{f}\left(\mathrm{x}_{1}\right)\right\rangle \geqslant 0\right\}=\mathrm{E} .
$$

Clearly $\Omega \subset \mathrm{Q}_{1}$. Suppose that $\Omega \subset \mathrm{Q}_{k}$ for some $k \in \mathbb{N}$. Then $\Omega \subset \mathrm{C}_{\mathrm{k}} \cap \mathrm{D}_{\mathrm{k}} \cap \mathrm{Q}_{\mathrm{k}}$. From $\mathrm{x}_{\mathrm{k}+1}=$ $P_{C_{k} \cap D_{k} \cap Q_{k}}^{f} x_{1}$, we have

$$
\left\langle x_{k+1}-p, \nabla f\left(x_{1}\right)-\nabla f\left(x_{k+1}\right)\right\rangle \geqslant 0, \quad \forall p \in C_{k} \cap D_{k} \cap Q_{k},
$$

then

$$
\left\langle x_{k+1}-p, \nabla f\left(x_{1}\right)-\nabla f\left(x_{k+1}\right)\right\rangle \geqslant 0, \quad \forall p \in \Omega .
$$

This implies $\Omega \subset Q_{k+1}$. Therefor by induction $\Omega \subset Q_{n}$ for all $n \geqslant 1$. Thus, we have $\Omega \subset C_{n} \cap D_{n} \cap Q_{n}$. This shows that $\left\{x_{n}\right\}$ is well defined.

Since by Lemma 3.1, F(G) and $F(T)$ are closed and convex, hence $\Omega$ is nonempty, closed and convex. This implies there exists $p \in \Omega$ such that $p=P_{\Omega}^{f} x_{1}$. Since $x_{n+1}=P_{C_{n} \cap D_{n} \cap Q_{n}}^{f} x_{1}$, from Lemma 2.1 and the fact that $p \in \Omega \subset C_{n} \cap D_{n} \cap Q_{n}$ for all $n \geqslant 1$ we have

$$
D_{f}\left(p, x_{n+1}\right)+D_{f}\left(x_{n+1}, x_{1}\right) \leqslant D_{f}\left(p, x_{1}\right),
$$

which implies

$$
D_{f}\left(x_{n+1}, x_{1}\right) \leqslant D_{f}\left(p, x_{1}\right) .
$$

This shows $\left\{D_{f}\left(x_{n}, x_{1}\right)\right\}$ is bounded, by Lemma 2.3, $\left\{x_{n}\right\}$ is bounded too. Since the functions $f$ and $g$ are bounded on bounded subsets of $E$ and therefore $\nabla f$ and $\nabla \mathrm{g}$ are also bounded on bounded subsets of $E^{*}$ this implies the sequences $\left\{\nabla f\left(x_{n}\right)\right\},\left\{\nabla f\left(y_{n}\right)\right\},\left\{\nabla f\left(z_{n}\right)\right\},\left\{\nabla g\left(B x_{n}\right)\right\}$ and $\left\{\nabla g\left(T B x_{n}\right)\right\}$ are bounded in $E^{*}$. have

Since $x_{n}=P_{Q_{n}}^{f} x_{1}$ and $x_{n+1}=P_{C_{n} \cap D_{n} \cap Q_{n}}^{f} x_{1}$, we have $x_{n+1} \in C_{n}$ for all $n \geqslant 0$. Using Lemma 2.1, we

$$
D_{f}\left(x_{n+1}, x_{n}\right)+D_{f}\left(x_{n}, x_{1}\right) \leqslant D_{f}\left(x_{n+1}, x_{1}\right)
$$

which implies

$$
D_{f}\left(x_{n}, x_{1}\right) \leqslant D_{f}\left(x_{n+1}, x_{1}\right),
$$

this shows that $\left\{D_{f}\left(x_{n}, x_{1}\right)\right\}$ is nondecreasing, consequently $\left\{D_{f}\left(x_{n}, x_{1}\right)\right\}$ is convergent. From Lemma 2.1 we have

$$
D_{f}\left(x_{n+1}, x_{n}\right)=D_{f}\left(x_{n+1}, P_{Q_{n}}^{f} x_{1}\right) \leqslant D_{f}\left(x_{n+1}, x_{1}\right)-D_{f}\left(P_{Q_{n}}^{f} x_{1}, x_{1}\right) \leqslant D_{f}\left(x_{n+1}, x_{1}\right)-D_{f}\left(x_{n}, x_{1}\right)
$$

for all $n \geqslant 1$. Which implies

$$
\lim _{n \rightarrow \infty} D_{f}\left(x_{n+1}, x_{n}\right)=0 .
$$

Since $f$ is totally convex on bounded subsets of $E, f$ is sequentially consistent (see [7]). It follows that

$$
\lim _{n \rightarrow \infty}\left\|x_{n+1}-x_{n}\right\|=0
$$

Again, from the fact that $x_{n+1}=P_{C_{n} \cap D_{n} \cap Q_{n}}^{f} x_{1}$, we have $x_{n+1} \in C_{n}$ for all $n \geqslant 0$

$$
\left\langle x_{n}-x_{n+1}, \nabla f\left(x_{n}\right)-\nabla f\left(z_{n}\right)\right\rangle \geqslant r_{n}(1-\eta) D_{g}\left(B x_{n}, T B x_{n}\right) .
$$


Since $\left\{r_{n}\right\}>0$, from (3.2) we have

$$
\lim _{n \rightarrow \infty} D_{g}\left(B x_{n}, T B x_{n}\right)=0 .
$$

Since $f$ is totally convex on bounded subsets of $E, f$ is sequentially consistent it follows that

$$
\lim _{n \rightarrow \infty}\left\|B x_{n}-T B x_{n}\right\|=0 .
$$

Since $\nabla f$ is norm-to-norm uniformly continuous on bounded subsets of $E^{*}$ we have

$$
\lim _{n \rightarrow \infty}\left\|\nabla g\left(B x_{n}\right)-\nabla g\left(\operatorname{TB} x_{n}\right)\right\|=0 .
$$

From (3.1) we have

$$
\left\|\nabla f\left(x_{\mathfrak{n}}\right)-\nabla f\left(z_{\mathfrak{n}}\right)\right\|=\left\|r_{n} B^{*}\left(\nabla g\left(B x_{\mathfrak{n}}\right)-\nabla g\left(T B x_{n}\right)\right)\right\| .
$$

Since $\left\{r_{n}\right\}$ is bounded, from (3.4) we have

$$
\lim _{n \rightarrow \infty}\left\|\nabla f\left(x_{n}\right)-\nabla f\left(z_{n}\right)\right\|=0 .
$$

Since $\nabla f$ is norm-to-norm uniformly continuous on bounded subsets of $E^{*}$ we have

$$
\lim _{n \rightarrow \infty}\left\|x_{n}-z_{n}\right\|=0 .
$$

But

$$
\left\|z_{n}-x_{n+1}\right\| \leqslant\left\|z_{n}-x_{n}\right\|+\left\|x_{n}-x_{n+1}\right\| .
$$

From (3.2) and (3.5) we have

$$
\lim _{n \rightarrow \infty}\left\|z_{n}-x_{n+1}\right\|=0 .
$$

Again, we have from $x_{n+1} \in D_{n}$ that

$$
\left\langle z_{n}-x_{n+1}, \nabla f\left(z_{n}\right)-\nabla f\left(y_{n}\right)\right\rangle \geqslant(1-\tau) D_{f}\left(z_{n}, y_{n}\right) .
$$

From (3.6) we have

$$
\lim _{n \rightarrow \infty} D_{f}\left(z_{n}, y_{n}\right)=0 \text {. }
$$

Since $f$ is totally convex on bounded subsets of $E, f$ is sequentially consistent, it follows that

$$
\lim _{n \rightarrow \infty}\left\|z_{n}-G z_{n}\right\|=0 .
$$

Since $\left\{x_{n}\right\}$ is bounded and the fact that $E$ is reflexive, there exists a sub-sequence $\left\{x_{n_{k}}\right\}$ of $\left\{x_{n}\right\}$ such that $x_{n_{k}} \rightarrow x^{*}$ as $k \rightarrow \infty$. From (3.5) there exists a sub-sequence $\left\{z_{n_{k}}\right\}$ of $\left\{z_{n}\right\}$ such that $z_{n_{k}} \rightarrow x^{*}$ as $k \rightarrow \infty$. From (3.7) and the assumption $G$ is demiclosed, we have $x^{*} \in F(G)$. Furthermore, since $B$ is a bounded linear map, we have $B x_{n_{k}} \rightarrow B x^{*}$ as $k \rightarrow \infty$. From demiclosedness of $T$ and (3.3) we have $B x^{*} \in F(T)$ which implies $x^{*} \in B^{-1} F(T)$. Hence $x^{*} \in \Omega$.

Finally, we show that $p=P_{\Omega} x_{0}$. With $p=P_{\Omega}^{f} x_{1}$ and $x^{*} \in \Omega$, we have

$$
D_{f}\left(p, x_{1}\right) \leqslant D_{f}\left(x^{*}, x_{1}\right),
$$

also, since $x_{n}=P_{\Omega}^{f} x_{1}$ and $p \in \Omega \subset C_{n} \cap D_{n} \cap Q_{n}$, we have

$$
D_{f}\left(x_{n}, x_{1}\right) \leqslant D_{f}\left(p, x_{1}\right) \text {. }
$$

Since $x_{n} \rightarrow x^{*}$, we have

$$
D_{f}\left(x^{*}, x_{1}\right) \leqslant D_{f}\left(p, x_{1}\right)
$$

From (3.8) and (3.9) we obtain that $D_{f}\left(x^{*}, x_{1}\right)=D_{f}\left(p, x_{1}\right)$. Thus $p=x^{*}=P_{\Omega}^{f} x_{1}$. This completes the proof. 
Corollary 3.3. Let $\mathrm{E}$ and $\mathrm{X}$ be uniformly convex and uniformly smooth real Banach spaces. Let $\mathrm{J}_{\mathrm{E}}$ and $\mathrm{J}_{\mathrm{X}}$ be the duality mappings on $\mathrm{E}$ and $\mathrm{X}$, respectively. Let $\eta$ and $\tau$ be a real numbers with $\eta, \tau \in(-\infty, 1)$. Let $\mathrm{G}: \mathrm{E} \rightarrow \mathrm{E}$ be $(\eta, 0)$ demigeneralized mapping with $\mathrm{F}(\mathrm{G}) \neq \emptyset$ and let $\mathrm{T}: \mathrm{X} \rightarrow \mathrm{X}$ be $(\tau, 0)$ demigeneralized mapping with $\mathrm{F}(\mathrm{T}) \neq \emptyset$. Let $\mathrm{B}: \mathrm{E} \rightarrow \mathrm{X}$ be a bounded linear operator such that $\mathrm{B} \neq 0$ and let $\mathrm{B}^{*}$ be the adjoint operator of $\mathrm{B}$. Suppose $\Omega=\mathrm{F}(\mathrm{G}) \cap \mathrm{B}^{-1} \mathrm{~F}(\mathrm{~T}) \neq \emptyset$. Let $\left\{\mathrm{x}_{\mathrm{n}}\right\}$ be a sequence generated by the following algorithm, and $\mathrm{x}_{1} \in \mathrm{E}$

$$
\left\{\begin{array}{l}
z_{n}=J_{E}^{-1}\left(J_{E} x_{n}-r_{n} B^{*}\left(J_{X} B x_{n}-J_{X} T B x_{n}\right)\right) \\
y_{n}=G z_{n} \\
C_{n}=\left\{y \in E:\left\langle x_{n}-y, J_{E} x_{n}-J_{E} z_{n}\right\rangle \geqslant r_{n}(1-\eta) \phi_{X}\left(B x_{n}, T B x_{n}\right)\right\} \\
D_{n}=\left\{y \in E:\left\langle z_{n}-y, J_{E} z_{n}-J_{E} y_{n}\right\rangle \geqslant(1-\tau) \phi_{E}\left(z_{n}, y_{n}\right)\right\} \\
Q_{n}=\left\{y \in E:\left\langle x_{n}-y, J_{E} x_{1}-J_{E} x_{n}\right\rangle \geqslant 0\right\} \\
x_{n+1}=\Pi_{C_{n} \cap D_{n} \cap Q_{n}} x_{1}, \quad n \geqslant 1,
\end{array}\right.
$$

where $r_{n} \subset(0, \infty)$ and $a \in \mathbb{R}$ satisfy the following inequalities

$$
0<\mathrm{a} \leqslant \mathrm{r}_{\mathrm{n}} \leqslant \frac{1}{\|\mathrm{~B}\|^{2}}, \quad \forall \mathrm{n} \in \mathbb{N} .
$$

Then $\left\{x_{n}\right\}$ converges strongly to $p \in \Omega$ and where $p=\Pi_{\Omega} x_{1}$.

\section{Applications}

Theorem 4.1. Let $\mathrm{E}$ and $\mathrm{X}$ be reflexive Banach spaces $\mathrm{f}: \mathrm{E} \rightarrow \mathbb{R}$ and $\mathrm{g}: \mathrm{X} \rightarrow \mathbb{R}$ are strongly coercive Legendre functions which are bounded uniformly Fréchet differentiable and totally convex on bounded subsets of $\mathrm{E}$ and $\mathrm{X}$, respectively. Let $\mathrm{G}: \mathrm{E} \rightarrow \mathrm{E}$ be quasi-Bregman nonexpansive mapping with $\mathrm{F}(\mathrm{G}) \neq \emptyset$ and let $\mathrm{T}: \mathrm{X} \rightarrow \mathrm{X}$ be quasiBregman strictly pseudocontractive mapping with $\mathrm{F}(\mathrm{T}) \neq \emptyset$. Let $\mathrm{B}: \mathrm{E} \rightarrow \mathrm{X}$ be a bounded linear operator such that $\mathrm{B} \neq 0$ and let $\mathrm{B}^{*}$ be the adjoint operator of $\mathrm{B}$. Suppose $\Omega=\mathrm{F}(\mathrm{G}) \cap \mathrm{B}^{-1} \mathrm{~F}(\mathrm{~T}) \neq \emptyset$, with the assumption that $\mathrm{T}$ is demiclosed. Let $\left\{x_{n}\right\}$ be a sequence generated by the following algorithm, and $x_{1} \in \mathrm{E}$

$$
\left\{\begin{array}{l}
z_{n}=\nabla f^{*}\left(\nabla f\left(x_{n}\right)-r_{n} B^{*}\left(\nabla g\left(B x_{n}\right)-\nabla g\left(T B x_{n}\right)\right)\right) \\
y_{n}=G z_{n}, \\
C_{n}=\left\{y \in E:\left\langle x_{n}-y, \nabla f\left(x_{n}\right)-\nabla f\left(z_{n}\right)\right\rangle \geqslant r_{n}(1-\eta) D_{g}\left(B x_{n}, T B x_{n}\right)\right\} \\
D_{n}=\left\{y \in E:\left\langle z_{n}-y, \nabla f\left(z_{n}\right)-\nabla f\left(y_{n}\right)\right\rangle \geqslant D_{f}\left(z_{n}, y_{n}\right)\right\} \\
Q_{n}=\left\{y \in E:\left\langle x_{n}-y, \nabla f\left(x_{1}\right)-\nabla f\left(x_{n}\right)\right\rangle \geqslant 0\right\} \\
x_{n+1}=P_{C_{n} \cap D_{n} \cap Q_{n}}^{f} x_{1}, \quad n \geqslant 1,
\end{array}\right.
$$

where $r_{n} \subset(0, \infty)$ and $a \in \mathbb{R}$ satisfy the following inequalities

$$
0<\mathrm{a} \leqslant \mathrm{r}_{\mathrm{n}} \leqslant \frac{1}{\|\mathrm{~B}\|^{2}}, \quad \forall \mathrm{n} \in \mathbb{N} .
$$

Then $\left\{x_{n}\right\}$ converges strongly to $p \in \Omega$ and where $p=\mathrm{P}_{\Omega}^{\mathrm{f}} \mathrm{x}_{1}$.

Proof. Since $T$ is quasi-Bregman strictly pseudocontractive mapping with $F(T) \neq \emptyset$, then $T$ is $(k, 0)$ Bregman demigeneralized mapping. Furthermore $T$ is democlosed from the assumption. Also we have quasi-Bregman nonexpansive mapping $\mathrm{G}$ is $(0,0)$-Bregman demigeneralized and demiclosed mapping. Therefore the result follows from Theorem 3.1.

Theorem 4.2. Let $\mathrm{E}$ and $\mathrm{X}$ be reflexive Banach spaces $\mathrm{f}: \mathrm{E} \rightarrow \mathbb{R}$ and $\mathrm{g}: \mathrm{X} \rightarrow \mathbb{R}$ are strongly coercive Legendre functions which are bounded uniformly Fréchet differentiable and totally convex on bounded subsets of $\mathrm{E}$ and $\mathrm{X}$, respectively. Let $\mathrm{U}$ and $\mathrm{A}$ be maximal monotone operators of $\mathrm{E}$ into $\mathrm{E}^{*}$ and $\mathrm{X}$ into $\mathrm{X}^{*}$, respectively. Let $\operatorname{Res}_{\lambda \mathrm{u}}^{\mathrm{f}}$ be the 
resolvent of $\mathrm{U}$ for $\lambda>0$ and let $\operatorname{Res}_{\mu \mathrm{A}}^{\mathrm{f}}$ be the resolvent of $\mathrm{A}$ for $\mu>0$. Let $\mathrm{B}: \mathrm{E} \rightarrow \mathrm{X}$ be a bounded linear operator such that $\mathrm{B} \neq 0$ and let $\mathrm{B}^{*}$ be the adjoint operator of $\mathrm{B}$. Suppose $\Omega=\mathrm{U}^{-1}(0) \cap \mathrm{B}^{-1}\left(\mathrm{~A}^{-1}(0)\right) \neq \emptyset$, with the assumption that $\operatorname{Res}_{\lambda \mathrm{U}}^{\mathrm{f}}$ and $\operatorname{Res}_{\mu \mathrm{A}}^{\mathrm{f}}$ are demiclosed. Let $\left\{x_{n}\right\}$ be a sequence generated by the following algorithm, and $x_{1} \in \mathrm{E}$

$$
\left\{\begin{array}{l}
z_{n}=\nabla f^{*}\left(\nabla f\left(x_{n}\right)-r_{n} B^{*}\left(\nabla g\left(B x_{n}\right)-\nabla g\left(\operatorname{Res}_{\mu A}^{f}\left(B x_{n}\right)\right)\right)\right) \\
y_{n}=\operatorname{Res}_{\lambda u}^{f}\left(z_{n}\right), \\
C_{n}=\left\{y \in E:\left\langle x_{n}-y, \nabla f\left(x_{n}\right)-\nabla f\left(z_{n}\right)\right\rangle \geqslant r_{n} D_{g}\left(B x_{n}, \operatorname{Res}_{\mu A}^{f}\left(B x_{n}\right)\right)\right\} \\
D_{n}=\left\{y \in E:\left\langle z_{n}-y, \nabla f\left(z_{n}\right)-\nabla f\left(y_{n}\right)\right\rangle \geqslant D_{f}\left(z_{n}, y_{n}\right)\right\} \\
Q_{n}=\left\{y \in E:\left\langle x_{n}-y, \nabla f\left(x_{1}\right)-\nabla f\left(x_{n}\right)\right\rangle \geqslant 0\right\} \\
x_{n+1}=P_{C_{n} \cap D_{n} \cap Q_{n}}^{f} x_{1}, \quad n \geqslant 1,
\end{array}\right.
$$

where $r_{n} \subset(0, \infty)$ and $a \in \mathbb{R}$ satisfy the following inequalities

$$
0<a \leqslant r_{n} \leqslant \frac{1}{\|B\|^{2}}, \quad \forall n \in \mathbb{N} .
$$

Then $\left\{x_{n}\right\}$ converges strongly to $\mathrm{p} \in \Omega$ and where $\mathrm{p}=\mathrm{P}_{\Omega}^{\mathrm{f}} \mathrm{x}_{1}$.

Proof. Since $\operatorname{Res}_{\lambda \mathrm{U}}^{\mathrm{f}}$ is the resolvent of $\mathrm{U}$ on $\mathrm{E}$ is $(0,0)$-Bregman demigeneralized mapping. Also since $\operatorname{Res}_{\mu A}^{f}$ is the resolvent of $A$ on $X$ is $(0,0)$-Bregman demigeneralized mapping. Furthermore $\operatorname{Res}_{\lambda u}^{f}$ and $\operatorname{Res}_{\mu A}^{f}$ are demiclosed. Therefore the result follows from Theorem 3.1.

Theorem 4.3. Let $\mathrm{E}$ and $\mathrm{X}$ be reflexive Banach spaces $\mathrm{f}: \mathrm{E} \rightarrow \mathbb{R}$ and $\mathrm{g}: \mathrm{X} \rightarrow \mathbb{R}$ are strongly coercive Legendre functions which are bounded uniformly Fréchet differentiable and totally convex on bounded subsets of $E$ and $X$, respectively. Let $\mathrm{G}: \mathrm{E} \rightarrow \mathrm{E}$ and $\mathrm{T}: \mathrm{X} \rightarrow \mathrm{X}$ be quasi-Bregman nonexpansive and demiclosed mappings, respectively. Let $\mathrm{B}: \mathrm{E} \rightarrow \mathrm{X}$ be a bounded linear operator such that $\mathrm{B} \neq 0$ and let $\mathrm{B}^{*}$ be the adjoint operator of $\mathrm{B}$. Suppose $\Omega=\mathrm{F}(\mathrm{G}) \cap \mathrm{B}^{-1} \mathrm{~F}(\mathrm{~T}) \neq \emptyset$. Let $\left\{\mathrm{x}_{\mathrm{n}}\right\}$ be a sequence generated by the following algorithm, and $\mathrm{x}_{1} \in \mathrm{E}$

$$
\left\{\begin{array}{l}
z_{n}=\nabla f^{*}\left(\nabla f\left(x_{n}\right)-r_{n} B^{*}\left(\nabla g\left(B x_{n}\right)-\nabla g\left(T B x_{n}\right)\right)\right) \\
y_{n}=G z_{n} \\
C_{n}=\left\{y \in E:\left\langle x_{n}-y, \nabla f\left(x_{n}\right)-\nabla f\left(z_{n}\right)\right\rangle \geqslant r_{n} D_{g}\left(B x_{n}, T B x_{n}\right)\right\} \\
D_{n}=\left\{y \in E:\left\langle z_{n}-y, \nabla f\left(z_{n}\right)-\nabla f\left(y_{n}\right)\right\rangle \geqslant D_{f}\left(z_{n}, y_{n}\right)\right\} \\
Q_{n}=\left\{y \in E:\left\langle x_{n}-y, \nabla f\left(x_{1}\right)-\nabla f\left(x_{n}\right)\right\rangle \geqslant 0\right\} \\
x_{n+1}=P_{C_{n} \cap D_{n} \cap Q_{n}}^{f} x_{1}, \quad n \geqslant 1,
\end{array}\right.
$$

where $r_{n} \subset(0, \infty)$ and $a \in \mathbb{R}$ satisfy the following inequalities

$$
0<\mathrm{a} \leqslant \mathrm{r}_{\mathrm{n}} \leqslant \frac{1}{\|\mathrm{~B}\|^{2}}, \quad \forall \mathrm{n} \in \mathbb{N}
$$

Then $\left\{x_{\mathrm{n}}\right\}$ converges strongly to $\mathrm{p} \in \Omega$ and where $\mathrm{p}=\mathrm{P}_{\Omega}^{\mathrm{f}} \mathrm{x}_{1}$.

Proof. Since $\mathrm{G}$ and $\mathrm{T}$ are quasi-Bregman nonexpansive mappings, $\mathrm{G}$ and $\mathrm{T}$ are $(0,0)$-Bregman demigeneralized mappings. Furthermore $G$ and $T$ are democlosed. Therefore the result follows from Theorem 3.1 .

\section{References}

[1] B. Ali, J. N. Ezeora, M. S. Lawan, Inertial algorithm for solving fixed point and generalized mixed equilibrium problems in Banach spaces, PanAmer. Math. J, 29 (2019), 64-83. 1

[2] E. Asplund, R. T. Rockafellar, Gradient of Convex Function, Trans. Amer. Math. Soc., 139 (1969), 443-467. 1 
[3] H. H. Bauschke, J. M. Borwein, Legendre Function and the Method of Bregman Projections, J. Convex Anal., 4 (1997), 27-67. 1

[4] H. H. Bauschke, P. L. Combettes, J. M. Borwein, Essential Smoothness, Essential Strict Convexity, and Legendre functions in Banach Spaces, Commun. Contemp. Math., 3 (2001), 615-647. 1.2, 1.3, 1, 3

[5] J. F. Bonnans, A. Shapiro, Perturbation analysis of optimization problems, Springer-Verlag, New York, (2000). 1, 1.3

[6] L. M. Bregman, The relaxation method of finding the common point of convex sets and its application to the solution of problems in convex programming, USSR Comput. Math. Phys., 7 (1967), 200-217. 1, 2

[7] D. Butnariu, A. N. Iusem, Totally Convex Functions for Fixed Points Computation and Infinite Dimensional Optimization, Kluwer Academic Publ., Dordrecht, (2000). 2.4, 2, 2, 3

[8] D. Butnariu, E. Resmerita, Bregman distances, totally convex functions, and a method for solving operator equations in Banach spaces, Abstr. Appl. Anal., 2006 (2006), 39 pages. 1, 2.1

[9] C. L. Byrne, Y. Censor, A. Gibali, S. Reich, Weak and strong convergence of algorithms for the split common null point problem, J. Nonlinear Convex Anal., 13 (2012), 759-775. 1

[10] Y. Censor, A. Lent, An Iterative row-action Method Interval Convex Programming, J. Optim. Theory Appl., 34 (1981), 321-353. 1

[11] Y. Censor, A. Segal, The split common fixed point problem for directed operators, J. Convex Anal., 16 (2009), 587-600. 1

[12] J.-B. Hiriart-Urruty, C. Lemaréchal, Convex analysis and minimization algorithms. II. Advanced theory and bundle methods, Springer-Verlag, Berlin, (1993). 1

[13] M. Hojo, W. Takahashi, A strong convergence theorem by shrinking projection method for the split null point problem Banach spaces, Numer. Funct. Anal. Optim., 37 (2016), 541-553. 1

[14] F. Kohsaka, W. Takahashi, Proximal point algorithms with Bregman functions in Banach spaces, J. Nonlinear Convex Anal., 6 (2005), 505-523. 2.5

[15] M. S. Lawan, B. Ali, M. H. Harbau, G. C. Ugwunnadi, Approximation of common fixed points for finite families of Bregman quasi-total asymptotically nonexpansive mappings, J. Nigerian Math. Soc., 35 (2016), 282-302. 1

[16] V. Martin-Marquez, S. Reich, S. Sabach, Right bregman nonexpansive operators in Banach space, Nonlinear Anal., 75 (2012), 5448-5465. 1

[17] E. Naraghirad, J. C. Yao, Bregman weak relatively nonexpansive mappings in Banach spaces, Fixed Point Theory Appl., 2013 (2013), 43 pages. 2.2

[18] S. Reich, S. Sabach, A strong convergence theorem for a proximal-type algorithm in reflexive Banach spaces, J. Nonlinear Convex Anal., 10 (2009), 471-485. 1.1

[19] S. Reich, S. Sabach, Two strong convergence theorems for a proximal method in reflexive Banach spaces, Numer. Funct. Anal. Optim., 31 (2010), 22-44. 2.3, 2.9

[20] S. Reich, S. Sabach, Two strong convergence theorems for Bregman strongly nonexpansive operators in reflexive Banach spaces, Nonlinear Anal., 73 (2010), 122-135. 1

[21] S. Reich, S. Sabach, Existence and approximation of fixed points of Bregman firmly nonexpansive mappings in reflexive Banach spaces, in: Fixed-point algorithms for inverse problems in science and engineering, 2011 (2011), 301-316. 1

[22] W. Takahashi, The split common fixed point problem and the hybrid method for demigeneralized mappings in two Banach spaces and application, J. Nonlinear Convex Anal., 18 (2017), 29-45. 1

[23] W. Takahashi, The split common null point problem for generalized resolvents in two Banach spaces, Numer. Algorithms, 75 (2017), 1065-1078. 1

[24] W. Takahashi, H.-K. Xu, J.-C. Yao, Iterative methods for generalized split feasibility problems in Hilbert spaces, Set-Valued Var. Anal., 23 (2015), 205-221. 1

[25] Y. Tomizawa, A strong convergence theorem for Bregman asymptotically quasi-nonexpansive mappings in the intermediate sense, Fixed Point Theory Appl., 2014 (2014), 14 pages.

[26] G. C. Ugwunnadi, B. Ali, I. Idris, M. S. Minjibir, Strong convergence theorem for quasi-Bregman strictly pseudocontractive mappings and equilibrium problems in Banach spaces, Fixed Point Theory Appl., 231 (2014), 1-16. 1, 2

[27] C. Zalinescu, Convex Analysis in General Vector Spaces, World Scientific Publishing Co., River Edge, (2002). 1, 2, 2 